\title{
The Partition Function for the Anharmonic Oscillator in the Strong-Coupling Regime
}

\author{
Nami Fux Svaiter* \\ Centro Brasileiro de Pesquisas Fisicas - CBPF \\ Rua Dr. Xavier Sigaud 150, Rio de Janeiro, RJ, 22290-180, Brazil \\ E-mail: nfuxsvai@cbpf.br
}

\begin{abstract}
We consider a single anharmonic oscillator with frequency $\omega$ and coupling constant $\lambda$ respectively, in the strong-coupling regime. We are assuming that the system is in thermal equilibrium with a reservoir at temperature $\beta^{-1}$. Using the strong-coupling perturbative expansion, we obtain the partition function for the oscillator in the regime $\lambda>>\omega$, up to the order $\frac{1}{\sqrt{\lambda}}$. To obtain this result, we use of a combination of Klauder's independent-value generating functional (Acta Phys. Austr. 41, 237 (1975)), and the spectral zeta function method. The free energy and the mean energy, up to the order $\frac{1}{\sqrt{\lambda}}$, are also presented.
\end{abstract}

Fourth International Winter Conference on Mathematical Methods in Physics 09 - 13 August 2004

Centro Brasileiro de Pesquisas Fisicas (CBPF/MCT), Rio de Janeiro, Brazil

\footnotetext{
* Speaker.
} 
The strong-coupling regime in quantum field theory is one of the unsolved problems of theoretical physics of the last century. There are many situations where one has to account for nonperturbative coupling regions, and to discuss the physics of the strongly coupled systems.

In this paper we present a method for calculating the partition function and the Helmholtz free energy for a single oscillator with the anharmonic $\frac{\lambda}{4 !} x^{4}(\tau)$ term in the strong-coupling regime up to the order $\frac{1}{\sqrt{\lambda}}$ [1]. For simplicity we are assuming that our system is one-dimensional and is in thermal equilibrium with a reservoir at temperature $\beta^{-1}$. We are working in the imaginary time formalism and making use of the Kubo-Martin-Schwinger (KMS) condition [2]. To find the partition function and the Helmholtz free energy, our approach consists in the combination of two techniques used currently in the literature: the strong-coupling expansion [3] [4] [5] [6] and the spectral zeta function method [7].

The basic idea of the strong-coupling expansion in approach in Euclidean field theory is the following: in a formal representation for the generating functional of complete Schwinger functions of the theory $Z(h)$, we treat the Gaussian part of the action as a perturbation with respect to the remaining terms of the functional integral. Therefore we are developing our perturbative expansion around the independent-value generating functional $Q_{0}(h)$, where different points of the Euclidean space are decoupled [8] [9] [10]. The fundamental problem of the strong-coupling expansion is how to give meaning to the independent-value generating functional. A naive use of a continuum limit of the lattice regularization for the independent-value generating functional leads to a Gaussian theory, where we simple make use of the central limit theorem. The fundamental modification which allow us to avoid the central limit theorem is a change in the measure in the functional integral [11] [12] [13].

Let us consider a one-dimensional quantum mechanical system. The partition function for the system assuming that it is in thermal equilibrium with a reservoir at temperature $\beta^{-1}$ is given by

$$
Z(\beta)=\int_{x(0)=x(\beta)}[d x(\tau)] \exp \left[-\int_{0}^{\beta} d \tau\left(\frac{1}{2} m\left(\frac{d x}{d \tau}\right)^{2}+V(x(\tau))\right)\right],
$$

where in the functional integral we require that $x(\tau)$ is periodic with period $\beta$, i.e., $x(\tau)=x(\tau+\beta)$. As usual, we define the generating functional $Z(\beta ; h)$ introducing an external source $h(\tau)$, and it is convenient to consider $h(\tau)$ to be pure imaginary. It is important to define the modified kernel $K\left(\omega, \sigma ; \tau-\tau^{\prime}\right)$ by the equation

$$
K\left(\omega, \sigma ; \tau-\tau^{\prime}\right)=\left(-\frac{d^{2}}{d \tau^{2}}+(1-\sigma) \omega^{2}\right) \delta\left(\tau-\tau^{\prime}\right)
$$

where $\omega$ is the frequency and $\sigma$ is a complex parameter defined in the region $0 \leq \operatorname{Re}(\sigma)<1$. The choice of a suitable $\sigma$ will simplify our calculations in some situations. For simplicity, we are choosing $m^{2}=1$.

To find the partition function for the anharmonic oscillator in the strong-coupling regime it is natural to use the strong-coupling perturbative expansion. We get the following formal representation for the generating functional at finite temperature $Z(\beta ; h)$ :

$$
Z(\beta ; h)=\exp \left(-\frac{1}{2} \int_{0}^{\beta} d \tau \int_{0}^{\beta} d \tau^{\prime} \frac{\delta}{\delta h(\tau)} K\left(\omega, \sigma ; \tau-\tau^{\prime}\right) \frac{\delta}{\delta h\left(\tau^{\prime}\right)}\right) Q(\beta, \sigma ; h),
$$


where $Q(\beta, \sigma ; h)$, the new independent-value functional integral, is given by

$$
Q(\beta, \sigma ; h)=\mathcal{N} \int_{x(0)=x(\beta)}[d x(\tau)] \exp \left[\int_{0}^{\beta} d \tau\left(-\frac{1}{2} \sigma \omega^{2} x^{2}(\tau)-\frac{\lambda}{4 !} x^{4}(\tau)+h(\tau) x(\tau)\right)\right],
$$

and the modified kernel $K\left(\omega, \sigma ; \tau-\tau^{\prime}\right)$ was defined by Eq.(2). The factor $\mathcal{N}$ is a normalization that can be found using that $\left.Q(\beta, \sigma ; h)\right|_{h=0}=1$. Note that we split the quadratic part in the functional integral, which is proportional to the frequency squared, into two parts; one contributes together with the derivative term in the action as the perturbation, and the other appears in the independentvalue generating functional. In the leading order, we have that $Z(\beta ; h)$ can be written as

$$
Z(\beta ; h)=\left(1-\frac{1}{2} \int_{0}^{\beta} d \tau \int_{0}^{\beta} d \tau^{\prime} \frac{\delta}{\delta h(\tau)} K\left(\omega, \sigma ; \tau-\tau^{\prime}\right) \frac{\delta}{\delta h\left(\tau^{\prime}\right)}\right) Q(\beta, \sigma ; h) .
$$

To evaluate $\ln Z(\beta ; h)$, note that we have two steps to follow. The first one is to give meaning to the independent-value generating-functional, and the second one is to regularize and renormalize the kernel $K\left(\omega, \sigma ; \tau-\tau^{\prime}\right)$ integrated over the volume $[0, \beta]$. Note that the parameter $\sigma$ was introduced only to simplify our calculations in some situations. Therefore $\sigma$ can be complex if we are able to work in all order of perturbation theory. The generating functional does not depends on the value for $\sigma$. Since we concentrate in the leading order, some care has to be taken to prevent a complex generating functional. A simple way to avoid the problem is assume that the parameter $\sigma$ is real. Therefore we will impose that $\operatorname{Im}(\sigma)=0$.

Since we are mainly interested in presenting the partition function, we can also assume that the external source is constant i.e. $h(\tau)=h$. Using the cumulant expansion idea, which relates the mean of a exponential to the exponential of means, after some simple calculations we obtain

$$
\ln Z(\beta ; h)=\frac{1}{Q(\beta, \sigma ; h)} \frac{\partial^{2} Q(\beta, \sigma ; h)}{\partial h^{2}}\left(-\frac{1}{2}+\left.\frac{1}{2} \frac{d}{d s} \zeta(s)\right|_{s=0}\right),
$$

where $\zeta(s)$ is the spectral zeta function associated with the operator $\left(-\frac{d^{2}}{d \tau^{2}}+(1-\sigma) \omega^{2}\right)$. This eliptic operator has a complete set of orthonormal eigenfunctions $x_{n}(\tau)$ and associated eigenvalues $a_{n}$. Using the boundary conditions $x_{n}(0)=x_{n}(\beta)$, we have that the spectral zeta function is given by

$$
\zeta_{-\frac{d^{2}}{d \tau^{2}}+(1-\sigma) \omega^{2}}(s)=\sum_{n=-\infty}^{\infty}\left[\left(\frac{2 \pi n}{\beta}\right)^{2}+(1-\sigma) \omega^{2}\right]^{-s} .
$$

Here, it is useful to define the Epstein-Hurwitz zeta function in the complex plane $s$, i.e., the function $\zeta(s, v)$ by:

$$
\zeta(s, v)=\sum_{n=-\infty}^{\infty}\left(n^{2}+v^{2}\right)^{-s}, \quad v^{2}>0
$$

Note that in the original Hurwitz zeta function it appears $n$ instead of $n^{2}$, while for the true Epstein zeta function no $n$-independent term should appear.

It is not difficult to write the spectral zeta function in terms of the Epstein-Hurwitz zeta function. We have

$$
\zeta_{-\frac{d^{2}}{d \tau^{2}}+(1-\sigma) \omega^{2}}(s)=\left(\frac{\beta}{2 \pi}\right)^{2 s} \zeta\left(s, \sqrt{1-\sigma}\left(\frac{\omega \beta}{2 \pi}\right)\right)
$$


where $\zeta(s, v)$, is the Epstein-Hurwitz zeta function. Using the analytic extention of the EpsteinHurwitz zeta function [14], is not difficult to show that

$$
\left.\zeta(s, v)\right|_{s=0}=0
$$

and

$$
\left.\frac{\partial}{\partial s} \zeta(s, v)\right|_{s=0}=-2 \ln (2 \sinh \pi v) .
$$

Since we are interested in calculating the derivative of the spectral zeta function at the origin of the complex $s$ plane, we have

$$
\left.\frac{1}{2} \frac{\partial}{\partial s} \zeta_{-\frac{d^{2}}{d \tau^{2}}+(1-\sigma) \omega^{2}}(s)\right|_{s=0}=\left.\left(\frac{1}{2} \zeta(s, v) \frac{d}{d s}\left(\frac{\beta}{2 \pi}\right)^{2 s}+\frac{1}{2}\left(\frac{\beta}{2 \pi}\right)^{2 s} \frac{\partial}{\partial s} \zeta(s, v)\right)\right|_{s=0} .
$$

Using our results in Eq.(10) we obtain

$$
\left.\frac{1}{2} \frac{\partial}{\partial s} \zeta_{-\frac{d^{2}}{d \tau^{2}}+(1-\sigma) \omega^{2}}(s)\right|_{s=0}=-\ln \left[\left(2 \sinh \left((1-\sigma) \frac{\omega \beta}{2}\right)\right] .\right.
$$

To complete our work we have to calculate the second derivative for the independent-value generating function with respect to the source. We would like to stress that we are using Klauder's result, as the formal definition of the independent-value generating functional derived for scalar fields in a $d$-dimensional Euclidean space. It is important to point out that in Klauder's derivation for the independent-value model a result was obtained which is well defined for all functions which are square integrable in $R^{n}$ i.e., $h(x) \varepsilon L^{2}\left(R^{n}\right)$. This observation allow us to conclude that we need also to use a normalization in the situation that we are investigating. It is possible to show that the independent-value generating function can be written as [11]

$$
Q(\beta, \sigma ; h)=\exp \left[-\frac{1}{2 \beta} \int_{0}^{\beta} d \tau \int_{-\infty}^{\infty} \frac{d u}{|u|}(1-\cos (h u)) \exp \left(-\frac{1}{2} \sigma \omega^{2} u^{2}-\frac{\lambda}{4 !} u^{4}\right)\right] .
$$

In order to study $Q(\beta, \sigma ; h)$, let us define $E(\omega, \sigma, \lambda ; h)$ given by

$$
E(\omega, \sigma, \lambda ; h)=\int_{-\infty}^{\infty} \frac{d u}{|u|}(1-\cos (h u)) \exp \left(-\frac{1}{2} \sigma \omega^{2} u^{2}-\frac{\lambda}{4 !} u^{4}\right) .
$$

Now let use the fact that the $\sigma$ parameter can be choosen in such a way that the calculations becomes tractable. Analysing only the independent-value generating functional it is not possible to write $Q(\beta, \sigma ; h)$ in a closed form even in the case of constant external source. One way to obtain a closed expression is to choose $\sigma=0$. Therefore, using a series representation for $\cos x$, we have

$$
\left.E(\omega, \sigma, \lambda ; h)\right|_{\sigma=0}=2 \sum_{k=1}^{\infty} \frac{(-1)^{k}}{(2 k) !} h^{2 k} \int_{0}^{\infty} d u u^{2 k-1} \exp \left(-\frac{\lambda}{4 !} u^{4}\right)
$$

At this point let us use the following integral representation for the Gamma function [15]

$$
\int_{0}^{\infty} d x x^{\nu-1} \exp \left(-\mu x^{p}\right)=\frac{1}{p} \mu^{-\frac{v}{p}} \Gamma\left(\frac{v}{p}\right), \operatorname{Re}(\mu)>0 \quad \operatorname{Re}(v)>0 \quad p>0 .
$$


Using the result given by Eq.(15) in Eq.(14) we have

$$
\left.E(\omega, \sigma, \lambda ; h)\right|_{\sigma=0}=\sum_{k=1}^{\infty} g(k) \frac{h^{2 k}}{\lambda^{\frac{k}{2}}},
$$

where $g(k)=\frac{1}{2} \frac{(-1)^{k}}{(2 k) !}(4 !)^{\frac{k}{2}} \Gamma\left(\frac{k}{2}\right)$. Substituting the Eq.(16) in Eq.(12) we obtain that the independentvalue generating function $\left.Q(\beta, \sigma ; h)\right|_{\sigma=0}$ can be written as

$$
\left.Q(\beta, \sigma ; h)\right|_{\sigma=0}=\exp \left[-\frac{1}{2 \beta} \int_{0}^{\beta} d \tau \sum_{k=1}^{\infty} g(k) \frac{h^{2 k}}{\lambda^{\frac{k}{2}}}\right] .
$$

It is easy to calculate the second derivative for the independent-value generating function with respect to $h$. Note that $\left.Q(\beta, \sigma ; h)\right|_{h=\sigma=0}=1$. Thus we have

$$
\left.\frac{\partial^{2} Q(\beta, \sigma ; h)}{\partial h^{2}}\right|_{h=\sigma=0}=\sqrt{\frac{3 \pi}{8 \lambda}} .
$$

Substituting the result obtained from the generalized zeta-function method given by Eq.(11) (choosing $\sigma=0)$ and Eq.(18) in Eq.(6) we have that $\ln Z(\beta)$ is given by

$$
\ln Z(\beta)=\sqrt{\frac{3 \pi}{8 \lambda}}\left[\frac{1}{2}-\ln \left(2 \sinh \left(\frac{\omega \beta}{2}\right)\right)\right] .
$$

Therefore the partition function for the single oscillator is

$$
Z(\beta)=\frac{e^{\frac{1}{2} \sqrt{\frac{3 \pi}{8 \lambda}}}}{\left(2 \sinh \left(\frac{\omega \beta}{2}\right)\right)^{\sqrt{\frac{3 \pi}{8 \lambda}}}} .
$$

Other thermodynamics quantities that we are able to find are the Helmholtz free energy and the mean energy. The Helmholtz free energy is given by

$$
F(\beta)=\sqrt{\frac{3 \pi}{8 \lambda}}\left[-\frac{1}{2 \beta}+\frac{\omega \beta}{2}+\frac{1}{\beta} \ln \left(1-e^{-\beta \omega}\right)\right] .
$$

Finally the mean energy is defined by $E=-\left.\frac{\partial}{\partial \beta} \ln Z(\beta)\right|_{h=0}$. Therefore we have

$$
E=\sqrt{\frac{3 \pi}{8 \lambda}}\left[\frac{\omega}{2}+\frac{\omega}{e^{\omega \beta}-1}\right] .
$$

The picture emerging from the previous discussion is the following: in the strong-coupling perturbative expansion we may split the problem of defining the generating functional into two parts: how to define precisely the independent-value generating functional and how to go beyond the independent-value approximation, taking into account the perturbation part. Our results show that the strong-coupling perturbative expansion, in combination with an analytic regularization procedure, is a useful method to compute global quantities, as the Helmholtz free energy, in the strong-coupling regime. 
In conclusion, in this article we studied the strong-coupling regime in one-dimensional models, after analytic continuation to imaginary time. One-dimensional models are very simple system for which we can apply our method in obtaining thermodynamics quantities in the leading order in the inverse of coupling constant. We calculate the partition function and the Helmholtz free energy for the anharmonic oscillator, using the strong-coupling perturbative expansion and the spectral zeta-function method. It was possible to present expressions up to the order $\frac{1}{\sqrt{\lambda}}$ for the partition function and the other thermodynamic quantities derived from the the Helmholtz free energy.

\section{References}

[1] We assume that the physical quantities are dimensionless. Consequently, it is convenient to introduce an arbitrary parameter $\mu$ with mass dimension to define all dimensionless physical quantities. For simplicity, we assume that $\mu=1$ since we are not interested in the scaling behavior of the model.

[2] R. Kubo, M. Toda and N. Hashitsume, Statistical Physics II, Springer Verlag, Berlin-Heidelberg (1991).

[3] S. Kovesi-Domokos, A Strong-Coupling Approximation: Covariant Perturbative Expansion Around Independent-Value Field Theories, Il Nuovo Cim. 33A (1976) 769.

[4] C. M. Bender, F. Cooper, G. S. Guralnik and D. H. Sharp, Strong-Coupling Expansion in Quantum Field Theory, Phys. Rev. D19 (1979) 1865.

[5] N. Parga, D. Toussaint and J. R. Fulco, Strong-Coupling Expansion in the Large N-Limit, Phys. Rev. D20 (1979) 887.

[6] N. F. Svaiter, The Strong-Coupling Expansion and the Ultra-Local Approximation in Field Theory, Physica A (2004), in press, [hep-th/0404070].

[7] S. W. Hawking, Zeta-Function Regularization of Path Integrals in Curved Spacetime, Comm. Math. Phys. 55 (1977) 133.

[8] G. Scarpeta and G. Vilasi, Spontaneous Breakdown of Symmetry in the Static Ultra-Local Approximation of the g $\lambda^{4}$ Quantum Field Theory, Il Nuovo Cim. 28A (1975) 62.

[9] H. G. Dosh Evaluation of Functional Integrals for an Ultralocal Static Field Theory, Nucl. Phys. $\mathbf{B 9 6}$ (1975) 525.

[10] R. Menikoff and D. H. Sharp, A Derivation of the Virial Expansion with Application to Euclidean Quantum Field Theory, J. Math. Phys. D14 (1976) 1952.

[11] J. R. Klauder, On Model Fields with Independent Values at Every Space-Time Point, Acta Phys. Aust. 41 (1975) 237.

[12] J. R. Klauder, Augmented Quantum Field Theory: A Proposal to Extend Conventional Formulations, Phys. Rev. D14 (1976) 1952.

[13] J. R. Klauder, New Measures for Non-Renormalizable Quantum Field Theory, Ann. Phys. 117 (1979) 19.

[14] L. H. Ford and N. F. Svaiter, One-Loop Renormalization of a Self-Interacting Scalar Field in Non-Simply Connected Spacetimes, Phys. Rev. 51 (1995) 6981.

[15] I. S. Gradshteyn and I. M. Ryzhik, Tables of Integrals, Series and Products, Academic Press Inc., New York (1980). 